\title{
New Concept of Telemetry X-Band Circularly Polarized Antenna Payload for CubeSat
}

\author{
Jamil Fouany, Marc Thevenot, Eric Arnaud, François Torres, Cyrille Menudier, Thierry Monediere \\ and Kevin Elis.
}

1

Abstract- This article presents a new concept of compact circularly polarized $\mathrm{X}$-Band $[8-8.4 \mathrm{GHz}]$ antenna for the $3 \mathrm{U}$ CubeSat platforms. Despite the integration constraints on the top face of the CubeSat, the design aims at an isoflux radiation pattern. This antenna associates a driving patch antenna and twelve parasitic crossed dipoles, both to minimize the axial ratio in the opening angle $\theta= \pm 65^{\circ}$ and to shape the radiation pattern. The patch excitation is carried out by a compact sequential-phase feed microstrip circuit. This antenna is manufactured and measured.

Index Terms - nano-satellite, circularly polarized X-band antenna, isoflux, parasitic dipoles, sequential-phase feed circuit.

\section{INTRODUCTION}

$\mathrm{T}$ HIS paper aims to propose a solution for a circularly polarized X-band antenna with a radiation pattern as close as possible to an isoflux coverage. The complexity of the challenge is to integrate the antenna on the upper face $(10 \mathrm{~cm} * 10 \mathrm{~cm})$ of a "3U" CubeSat platform. This study has been carried out within the framework of a CNES (French space agency) Research and Technology (R\&T) program. This development led to a prototype, thus achieving a Technology Readiness Level (TRL) of 3.

The particularity of the CubeSats is the shape and size standardization that make them today the most popular of all nanosats [1]. These platforms are composed of a stack of elementary volumes $(10 \mathrm{~cm} * 10 \mathrm{~cm} * 10 \mathrm{~cm})$, named " $1 \mathrm{U}$ ". A $3 \mathrm{U}$ CubeSat looks like a parallelepiped of $10 \mathrm{~cm} * 10 \mathrm{~cm} * 30 \mathrm{~cm}$, which allows it to be launched with a P-Pod deployer [1]. The CubeSats and their payloads benefit of low cost and short development, making them very attractive as technology test and demonstration platforms in order to limit risks in future missions. These platforms mainly interest the academics (training), the industry (Technical demonstration and technology) and the government space agencies. Applications of Nanosats include high data rate telemetry, observation, scientific payloads, high-resolution still imaging, maritime applications such as ship tracking [2]... Most of these missions require an on-board VHF or S-band quasi isotropic

Manuscript received July 2017

Jamil FOUANY, Marc THEVENOT, Eric ARNAUD, François- TORRES, Cyrille MENUDIER and Thierry MONEDIERE are with the university of Limoges, XLIM-CNRS UMR 7252, 87060 Limoges, France (e-mail: marc.thevenot@xlim.fr).

Kevin ELIS is with the CNES (French space agency),18 Avenue Edouard Belin, 31401 Toulouse Cedex 9, France. (e-mail: Kevin.Elis@cnes.fr). antenna for sat-to-sat or sat-to-earth communications, but VHF links have moderate capability (low data rate) due to the narrow bandwidth. To enhance the data rate communication for future missions, it appears useful to investigate higher frequencies offering larger bandwidths.

Our work focuses on the $\mathrm{X}$ band to establish a high data rate downlink with Earth. For this purpose, a high-gain base station beam-steering antenna should be used for the LEO nanosat tracking. Therefore, an antenna payload having an ideally isoflux radiation pattern will offer the longer visibility time from the base station. In the context of the CubeSat, the EIRP is limited by the low available RF power $(<2$ Watt on $3 \mathrm{U}$ CubeSat platforms) and the large radiation coverage required by this future mission (isoflux antenna). For this reason, the antenna must offer both good radiation efficiency and good circular polarization over all directions.

The antenna proposed in this paper is designed to be integrated on the $10 \mathrm{~cm} * 10 \mathrm{~cm}$ square face of $3 \mathrm{U}$ CubeSat. The antenna is compact and thin enough to be compatible with $\mathrm{P}$ Pod launcher. The French space agency (CNES) provided specifications presented in table I.

TABLE I

ANTENNA SPECIFICATIONS

\begin{tabular}{ll}
\hline \multicolumn{1}{c}{ Parameters } & \multicolumn{1}{c}{ Specifications } \\
\hline Frequency Band $(\mathrm{GHz})$ & X-band $[8.0-8.4 \mathrm{GHz}]$ \\
Return Loss $(\mathrm{dB})$ & $<-20 \mathrm{~dB}$ \\
Polarization & $\mathrm{RHCP}$ \\
Limit of Coverage & $\theta=65^{\circ}$ \\
Minimum Gain & $0 \mathrm{dBi}$ \\
Radiation Pattern & Isoflux (if possible) \\
Axial Ratio (dB) & $<3 \mathrm{~dB}$ in the opening angle $\theta= \pm 65^{\circ}$ \\
Antenna Dimensions & Footprint $=9 \mathrm{~cm} * 9 \mathrm{~cm}$ \\
& Thickness $<9 \mathrm{~mm}$ outside the Satellite \\
Admissible RF Power & $2 \mathrm{Watt}$ \\
\hline \hline
\end{tabular}

A recent paper about the antenna developments for CubeSats [3] shows that most of antennas are designed for VHF or S bands, and only a few antenna concepts are intended for $\mathrm{X}$ band. Among these developments, a directive patch antenna array is proposed in [4], a reflectarray in [5] and a large deployable antenna for SAR systems in [6]. All these antennas are high-gain ones and cannot meet our specifications. In the context of our project, two interesting new developments for CubeSat have explored a miniaturized helix [7] and an EBG Matrix antenna [8] to radiate the isoflux diagram, and our work proposes an alternative to these designs. 
The main challenge of this project is to integrate a high quality circularly polarized isoflux X-band antenna on top of the $10 \mathrm{~cm} * 10 \mathrm{~cm}$ square earth-face of $3 \mathrm{U}$ CubeSat. The antenna families known to meet these requirements are the quadrifilar helix antenna [9] and the choke horn antenna [10]. Unfortunately, the former is more than one wavelength high [11], and the aperture diameter of the latter is greater than ten wavelengths, which is incompatible with our integration requirements. Two other original solutions able to produce isoflux radiation patterns have been studied in the literature: one uses metasurfaces [12] and the other is a slots array fed by the radial mode of a planar waveguide [13]. Again, both solutions are too large for CubeSats.

This letter presents the complete design, realization and measurements of a new antenna which uses parasitic elements for complying with the radiation specifications and the integration requirements.

\section{Summary OF the ANTENNA PREvious DESIGN}

This work refers to the previous general design we published in [14]. We give readers a brief reminder of the antenna conception and the simulated electromagnetic properties. The principle of this antenna is to associate a sequential rotation phase shift driving a patch antenna with a set of parasitic crossed dipoles. These dipoles are used for maximizing the gain and the axial ratio in the opening angle $\pm 65^{\circ}$ [14]. The antenna architecture shown in Fig. 1 was optimized to comply with an integration footprint on top the $10 \mathrm{~cm} \times 10 \mathrm{~cm}$ upper face of the $3 \mathrm{U}$ CubeSats. It is composed of a circularly polarized patch antenna connected to a compact sequential-phase feed microstrip circuit. This circuit is made up of one oversized $180^{\circ}$ hybrid ring coupler and two $90^{\circ}$ hybrid couplers, which are folded to fit inside the $180^{\circ}$ ring coupler. The patch and the circuit are printed on two stacked RO4003c substrates, creating a buried ground plane between the patch and the couplers' board. The patch antenna is connected to the couplers through four via holes crossing the buried ground plane. The resulting microstrip assembly is placed on a metal cylinder $(13 \mathrm{~mm}$ high and $33 \mathrm{~mm}$ in diameter), which is surrounded by twelve parasitic crossed dipoles placed on a $45 \mathrm{~mm}$-diameter circle, printed on both sides of a second $\mathrm{RO} 4003 \mathrm{c} 1.524 \mathrm{~mm}$-thick substrate. Interactions with the set of crossed dipoles were optimized using the method published in [15] and [16] which has been improved to deal with circular polarization. The method solves reactive loads that must be connected to the dipoles in order to meet the radiation objectives (both diagram shaping and polarization). For right-handed circular polarization, the optimization leads to reactive functions different for the dipoles printed on the upper face and the ones on the back face (crossed dipoles). These reactive functions can be emulated by adjusting the lengths and the gaps of the dipoles. Therefore, the six dipoles that are printed on the upper face of the substrate are $0.8 \mathrm{~mm}$ wide, $11.7 \mathrm{~mm}$ long, with a $0.5 \mathrm{~mm}$ gap. The other six dipoles printed on the back are short-circuited (gap $=0$ ), they are $12.3 \mathrm{~mm}$ long and $0.8 \mathrm{~mm}$ wide. This second substrate (printed parasitic dipoles) lies $1.5 \mathrm{~mm}$ above $9.5 \mathrm{~mm}$ deep concentric corrugations (Fig. 1). These corrugations both weaken the surface currents and forbid the possible cavity resonances (the ground plane which is set below the upper face of the nanosat forms an open cylindrical cavity). The antenna assembly thickness is $15 \mathrm{~mm}$ and only $7.5 \mathrm{~mm}$ exceed the upper face of the platform. The radiation performances simulated in [14] are recalled: for theta varying from $0^{\circ}$ to $+60^{\circ}$ the radiation pattern is not isoflux but the gain is always greater than $0.4 \mathrm{dBi}$; on the other hand the axial ratio stays below $2.5 \mathrm{~dB}$ whatever the radiation direction. The return loss simulated with numerical waveguide ports used as matched terminations is lower than $-19 \mathrm{~dB}$. This antenna preliminary design fulfills the needs over the entire frequency bandwidth.

Fig. 2 illustrates the contribution of the parasitic dipoles to the radiation optimization: the dipoles increase the gain by more than $1 \mathrm{~dB}$ at theta $60^{\circ}$ and the axial ratio is reduced over a wide aperture angle.

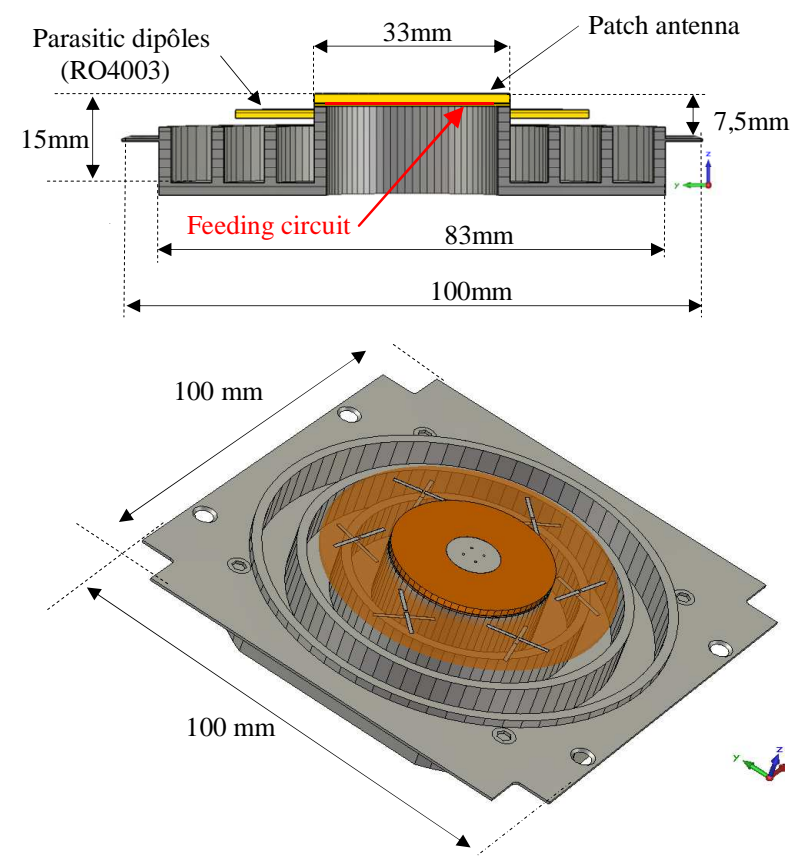

Fig. 1. Antenna design with parasitic crossed dipoles

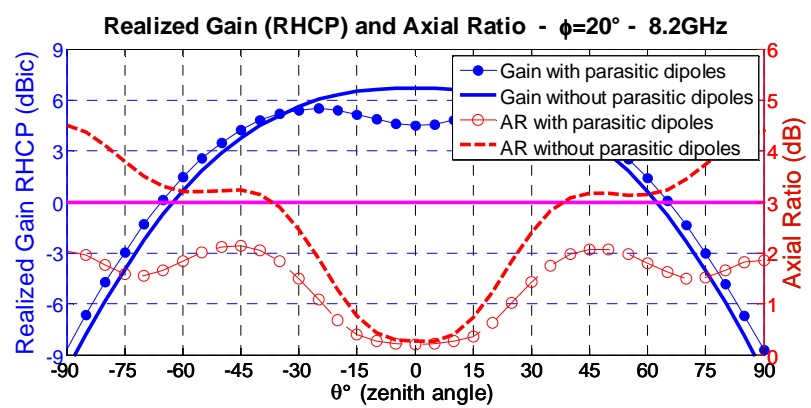

Fig. 2. Demonstration of the parasitic dipoles effects on the radiation pattern and axial ratio (simulations) - plane $\phi=20^{\circ}$ - freq $=8.2 \mathrm{GHz}$

\section{DESIGN FINALIZATION}

The design method used for the antenna conception was presented in [14], and will not be recalled here. However the circuit is now completed by realistic terminations. The numerical waveguide ports used for the simulation are changed into lumped $50 \Omega$ resistors and a coaxial cable is 
connected at the input of the circuit. Two different coplanarto-microstrip transitions are optimized to receive the $50 \Omega$ 0402-SMD resistors [17] and the "UT-047" semi-rigid coaxial cable (1.19mm in diameter). The circuit drawing including the coaxial cable and the SMD resistors is presented in Fig. 3. A simulation of this whole circuit confirmed the performances simulated without the physical terminations (in [14]). The magnitude balance is less than $0.2 \mathrm{~dB}$ between the four patch feeding probes and the maximum phase error between two adjacent feeding probes (via holes $0.5 \mathrm{~mm}$ in diameter) is about $2^{\circ}$ over the frequency bandwidth.

The return loss of the antenna is shown in Fig. 4. We can see that the return loss of circuit alone [14] (pink curve) is slightly degraded by the coaxial transition (black curve). The return loss of the whole circuit with the SMD resistors and the coaxial cable is higher than $-13 \mathrm{~dB}$ over $[8-8.4 \mathrm{GHz}]$ whereas it was $-19 \mathrm{~dB}$ for the circuit terminated by numerical waveguide ports (from [14]). The patch active return loss (sequential-phase feed) reaches $-11 \mathrm{~dB}$ at $8 \mathrm{GHz}$ and $-9 \mathrm{~dB}$ at $8.4 \mathrm{GHz}$ (Fig. 4), these values will degrade the antenna radiation efficiency.

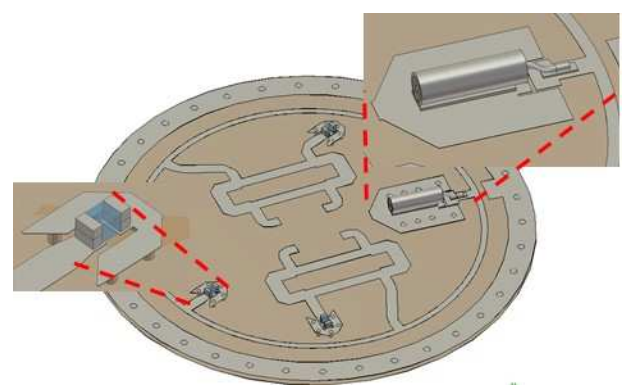

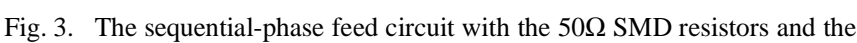
coaxial connector

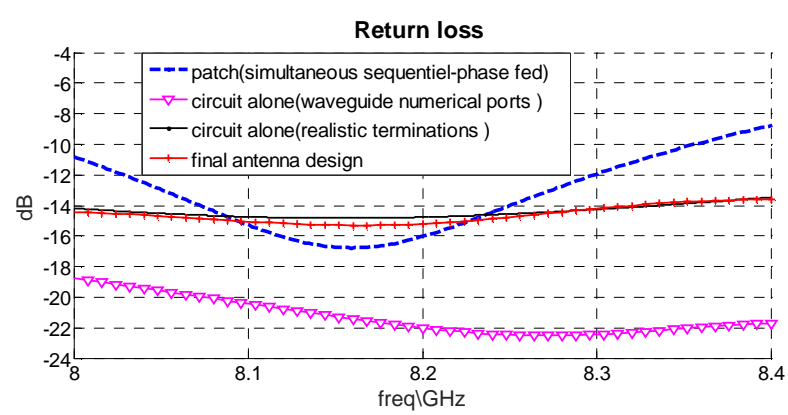

Fig. 4. Return loss at different steps in the antenna design (simulations)

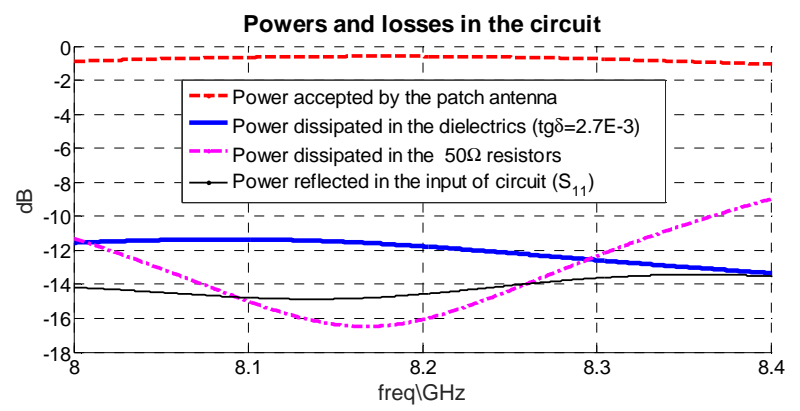

Fig. 5. Power and losses in the circuit (simulation)
The circuit losses are analyzed in Fig. 5. The circuit dielectric losses are around $0.3 \mathrm{~dB}$ (dissipated power is less than $-11 \mathrm{~dB}$ ). By design, powers reflected by the patch are dissipated in the $50 \Omega$ resistors, and the total lost power is lower than $-10 \mathrm{~dB}(-9 \mathrm{~dB}$ at $8.4 \mathrm{GHz})$. Finally, we evaluate the total efficiency of the simulated sequential-phase feed microstrip circuit to be around $-1 \mathrm{~dB}$.

\section{REALIZATION AND MEASUREMENTS}

The different parts of the antenna have been manufactured and put together. The patch antenna and the sequential-phase feed circuit are printed on two RO4003c substrates $\left(\varepsilon_{\mathrm{r}}=3.55\right.$, $\operatorname{tg} \delta=2.7 \mathrm{E}-3$ ). The $1.524 \mathrm{~mm}$-thick patch substrate is stacked with the $0.406 \mathrm{~mm}$-thick feeding circuit. Connections between circuit and patch are four $0.5 \mathrm{~mm}$ metallized via holes. The upper and lower sides of this circularly polarized driving patch antenna are shown in Fig. 6 (right). Fig. 7 proposes a view of the coaxial cable connecting the circuit and the SMD resistors [17] that are soldered on the optimized terminations. The parasitic crossed dipoles are printed on both sides of a second $1.524 \mathrm{~mm}$ thick RO4003c substrate (Fig. 6, left). For the measurements, the assembled antenna is finally set up on a 3U-CubeSat platform (Fig. 8).
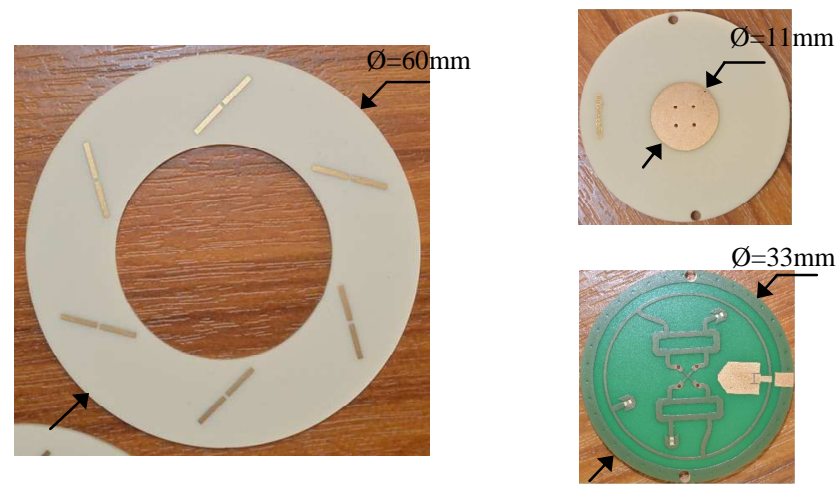

Fig. 6. Parasitic dipoles are printed on both sides of RO4003c substrate (left) - The patch antenna is printed on the 2 layers assemby and the sequentialphase feed circuit is printed backside (right)
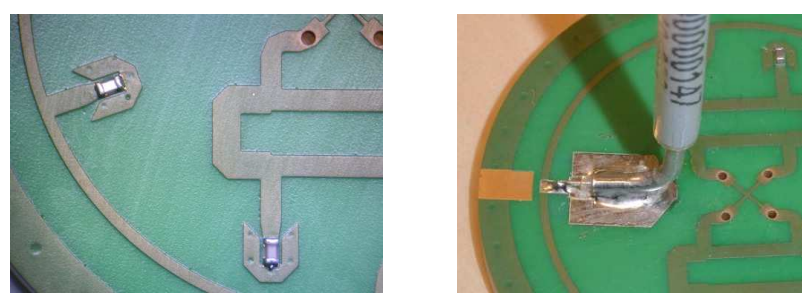

Fig. 7. SMD components and coaxial cable are soldered on the circuit.

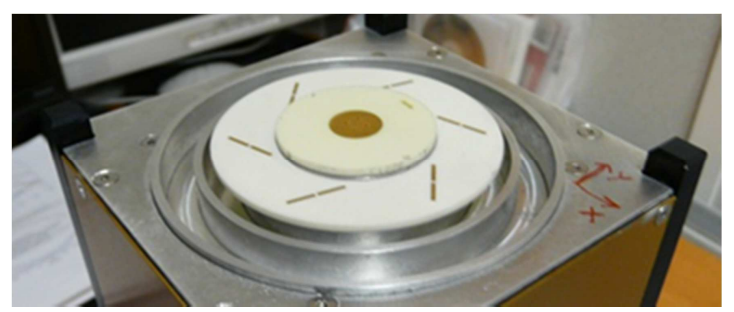

Fig. 8. The manufactured antenna is integrated on top of a $3 \mathrm{U}$ platform 
The measured realized gain is plotted at $8.2 \mathrm{GHz}$ for $\phi=0^{\circ}$ and $\phi=45^{\circ}$, and these plots are compared with simulations (Fig. 9 and Fig. 10). The gain is $-0.4 \mathrm{dBi}$ at $\theta=60^{\circ}$, which is $0.8 \mathrm{~dB}$ lower than the simulation and indicates higher losses in PCBs. The cross-polarization discrimination is greater than $15 \mathrm{~dB}$ for $\theta$ varying between $-60^{\circ}$ and $+60^{\circ}$. The measured axial ratio (plotted in Fig. 11 and Fig. 12) meets the specifications $(<3 \mathrm{~dB})$ from 8 to $8.4 \mathrm{GHz}$ and agrees with the simulation. Due to the symmetries in the antenna design, the radiation patterns are assumed to remain unchanged for any cutting plane over the entire frequency band. This expected omni-directivity is assessed in Fig. 13: the gain and axial ratio plotted in azimuth planes at three elevation angles $\left(\theta=30^{\circ}, 50^{\circ}\right.$ and $60^{\circ}$ ) confirm the omnidirectionality of the manufactured antenna.

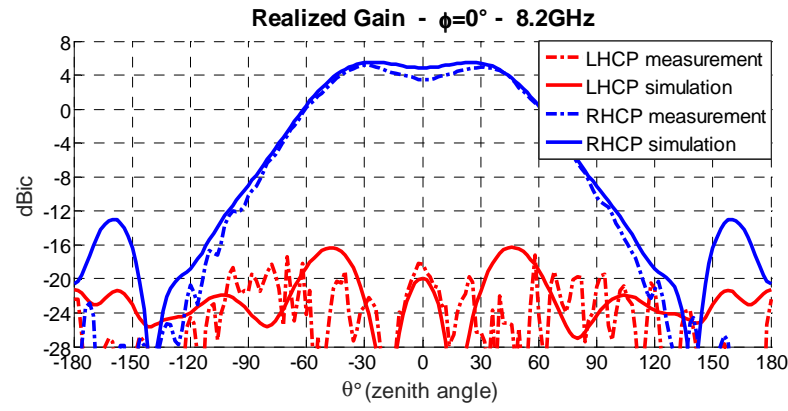

Fig. 9. Measured and simulated realized gain - plane $\phi=0^{\circ}$

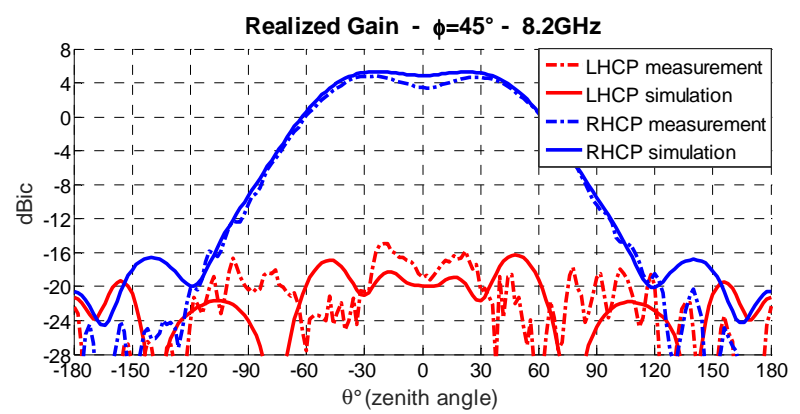

Fig. 10. Measured and simulated realized gain - plane $\phi=45^{\circ}$

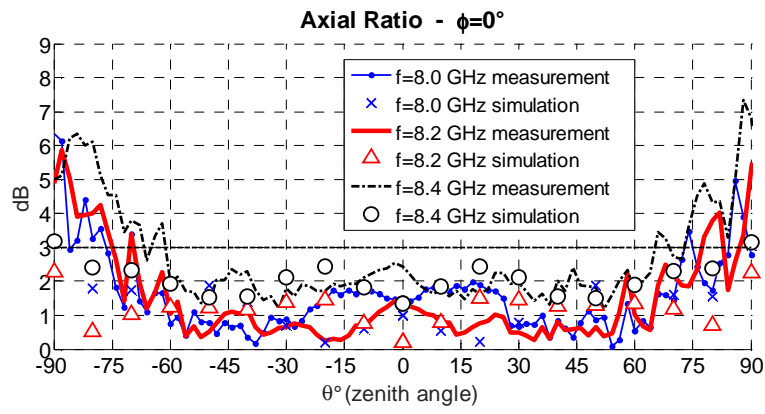

Fig. 11. Measured and simulated axial ratio - plane $\phi=0^{\circ}$

The measured antenna return loss (Fig. 14) is lower than $-10 \mathrm{~dB}$. The antenna is well matched even if there is a small difference between measurement and simulation (Fig. 4) which can be explained by the coaxial cable transition assembly. From Fig. 14, the measured radiation efficiency is about $70 \%$ whereas the simulated one is over $80 \%$. This difference is explained by a possible mismatching between the patch and the circuit. In such a case the reflected power is dissipated in the $50 \Omega$ resistors.

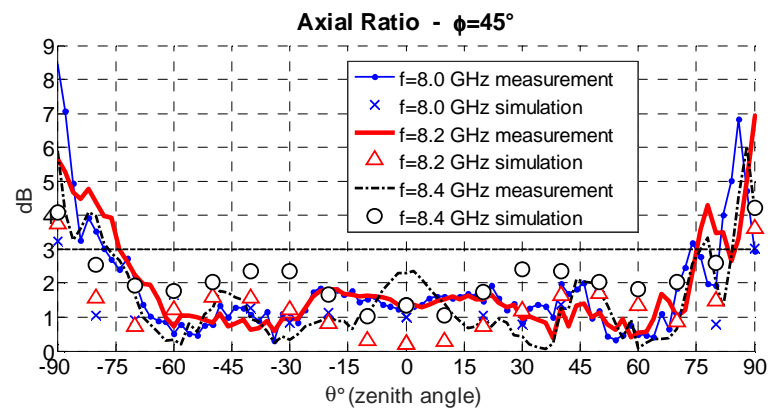

Fig. 12. Measured and simulated axial ratio - plane $\phi=45^{\circ}$

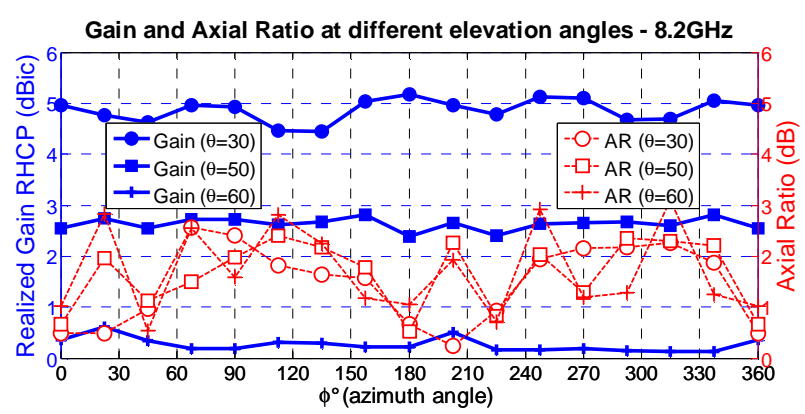

Fig. 13. Measured gain and axial ratio at 3 elevation angles

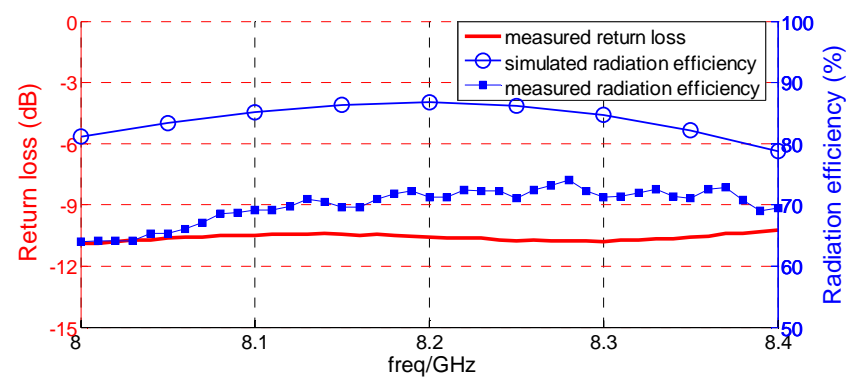

Fig. 14. Measured return loss and radiation efficiency

\section{CONCLUSION}

This paper demonstrates the performances of an original $\mathrm{X}$ band circularly polarized antenna developed for integration on the top face of a $3 \mathrm{U}-\mathrm{CubeSat}$. The antenna is $15 \mathrm{~mm}$ thick with only $7.5 \mathrm{~mm}$ outside the satellite. The exact isoflux radiation objective could not be achieved due to the platform size yet the maximum gain is at theta $30^{\circ}$ and it reaches $0 \mathrm{~dB}$ at $60^{\circ}$. The minimization of the axial ratio in the opening angle $\pm 65^{\circ}$ was carried out by introducing parasitic crossed dipoles. The measured radiation efficiency is about $70 \%$. All the measurements agree with the simulations except for the return loss, which suffer from a defective connector. This new design is a laboratory prototype, and can still be upgraded. A more efficient connector and a wider bandwidth patch would improve the antenna efficiency.

The authors would like to thank the French Space Agency (CNES) which funded this study. 


\section{REFERENCES}

[1] ARC Mission Design Division Staff, "Small spacecraft technology state of the art," NASA Ames Research Center, Moffet Field, CA, Tech. Rep. NASA/TP-2014-216648/REV1, 2014.

[2] Rainee N. Simons, "Applications of Nano-Satellites and CubeSatellites," Microwave Symposium (IMS), 2015 IEEE MTT-S International, Phoenix, AZ, USA, July 2015

[3] Yahya Rahmat-Samii, Vignesh Manohar, and Joshua M. Kovitz, "For Satellites, Think Small, Dream Big," IEEE Antennas \& Propagation Magazine, Volume: 59, Issue: 2, April 2017.

[4] Thomas J. Mizuno, Justin D. Roque, Blaine T. Murakami, Lance K. Yoneshige, Grant S. Shiroma, Ryan Y. Miyamoto, and Wayne A Shiroma, "Antennas for Distributed Nanosatellite," Wireless Communications and Applied Computational Electromagnetics, IEEE/ACES, International Conference on 3-7 April 2005, USA

[5] Richard E. Hodges, Daniel J Hoppe, Matthew J Radway, Nacer E. Chahat, "Novel Deployable Reflectarray Antennas for CubeSat Communications," Microwave Symposium (IMS), 2015 IEEE MTT-S International, Phoenix, AZ, USA, July 2015

[6] Prilando Rizki Akbar, Hirobumi Saito Miao Zang, Jiro Hirokawa, Makoto Ando, "X-Band Parallel-Plate Slot Array Antenna for SAR Sensor onboard $100 \mathrm{~kg}$ Small Satellite," Antennas and Propagation \& USNC/URSI National Radio Science Meeting, 2015 IEEE International Symposium on 19-24 July 2015 Vancouver, BC, Canada

[7] J Rodrigo Manrique, Gwenn Le Fur, Nicolas Adnet, Luc Duchesne, Jean Marc Baracco, Kevin Elis, "Telemetry X-band Antenna Payload for Nano-satellites," Antennas and Propagation (EUCAP), 2017 11th European Conference on 19-24 March 2017, Paris france

[8] Ali Siblini , Hussein Abou Taam , Bernard Jecko, Mohamed Rammal, Anthony Bellion, "New Agile EBG Matrix Antenna for Space Applications," Microwave Conference (EuMC), 2016 46th European, 46 Oct. 2016, london

[9] Dante Colantonio, Claus Rosito, A Spaceborne, "Telemetry Loaded Bifilar Helical Antenna for LEO Satellites," Antenna Group Technology Transfer Division. Villa Elisa, Buenos Aires, 1894, Argentina.

[10] Ravanelli R., Iannicelli C., Baldecchi N., Franchini F., "Multi-objective optimization of an isoflux antenna for LEO satellite down-handling link," Microwave Radar and Wireless Communications (MIKON), 2010 18th International Conference on, vol., no., pp.1, 4, 14-16 June 2010.

[11] C. Kilgus Johns Hopkins, "Shaped-Conical Radiation Pattern Performance of the Backfire Quadrifilar Helix", IEEE Transactions on Antennas and Propagation, Volume: 23, Issue: 3, May 1975

[12] Gabriele Minatti, Stefano Maci, Fellow, Paolo De Vita, Angelo Freni and Marco Sabbadini, "A Circularly-Polarized Isoflux Antenna Based on Anisotropic Metasurface," IEEE Transactions on Antenna and Propagation, VOL. 60, NO. 11, November 2012.

[13] Matteo Albani, Agnese Mazzinghi, and Angelo Freni, "Automatic Design of CP-RLSA approach," IEEE Transactions on Antenna and Propagation, VOL. 60, NO. 12, December 2012.

[14] J.Fouany, M.Thevenot, E. Arnaud, F.Torres , T.Monediere, N.Adnet, R. Manrique, L. Duchesne, J.M. Baracco K. Elis, "Circurlaly polarized isoflux compact X band antenna for nano-satellites applications," Radar Conference (EuRAD), 2015 European 9-11 Sept. 2015, Paris, France.

[15] M. Thevenot, C. Menudier, A. El Sayed Ahmad, G. Zakka El Nashef, F. Fezai, Y. Abdallah, E. Arnaud, F. Torres, and T. Monediere, "Synthesis of Antenna Arrays and Parasitic Antenna Arrays with Mutual Couplings," Int. J. Antennas Propag., vol. 2012, Article ID 309728.

[16] Faycel.Fezai, Cyrille.Menudier, Marc Thevenot and Thierry. Monédière, "Systematic Design of Parasitic Element Antennas Application to WLAN Yagi Design," IEEE Antennas And Wirless Propag Letters, VOL. 12, 2013

[17] http://www.atceramics.com/UserFiles/504L_ubr_resistor.pdf 